\title{
Atypical presentation of complex regional pain syndrome: neuropathic itching - A case report -
}

\section{Syn-Hae Yoon ${ }^{1}$, Woojong $\mathrm{Cho}^{2}$, Juhan Mun ${ }^{2}$, Wonyeong Jeong ${ }^{2}$, Young Do Kim ${ }^{2}$, and Seong-Soo $\mathrm{Choi}^{2}$}

'Department of Anesthesiology and Pain Medicine, National Police Hospital, Seoul, Korea ${ }^{2}$ Department of Anesthesiology and Pain Medicine, Asan Medical Center, University of Ulsan College of Medicine, Seoul, Korea

Received January 17, 2021

Revised July 6, 2021

Accepted July 26, 2021

\section{Corresponding author}

Seong-Soo Choi, M.D., Ph.D.

Department of Anesthesiology and

Pain Medicine, Asan Medical Center,

University of Ulsan College of

Medicine, 88 Olympic-ro 43-gil,

Songpa-gu, Seoul 05505, Korea

Tel: 82-2-3010-1538

Fax: 82-2-3010-6790

E-mail: choiss@amc.seoul.kr

Current affiliation: Syn-Hae Yoon is now with Department of Anesthesiology and Pain Medicine, El Hospital, Namyangju, Korea.

Previous presentation in conferences: This work was partly presented in the American Society of Anesthesiologists Annual Meeting (2017).
Background: In some patients with neuropathic pain (NP), such as complex regional pain syndrome (CRPS), itching rather than pain is the main symptom making diagnosis and treatment difficult.

Case: We report a case of a 23-year-old male with a history of hypoxic brain damage who presented with pruritus of the left foot and ankle. His left foot was fractured, and he underwent surgery 6 months previously. After the operation and cast application, he developed uncontrolled pruritus, swelling, sweating, and flushing of the left foot skin with limping. On examination, he showed well-known features of CRPS without pain. He was diagnosed with an atypical CRPS with neuropathic itching (NI). With treatment modalities used for NP and CRPS, his pruritus subsided gradually, and the his ankle mobility improved.

Conclusions: Unexplained itching can be the main symptom in some CRPS patients. Treatment according to NP can improve symptoms of NI in CRPS patients.

Keywords: Complex regional pain syndrome; Fracture; Itch; Neuropathic itch; Neuropathic pain.
Complex regional pain syndrome (CRPS) is a painful disorder, characterized by pain following an inciting injury and a variable convergence of signs and symptoms of sensory, vasomotor, sudomotor, trophic, and motor dysfunction [1]. CRPS appears to be caused by various components of inflammatory factors, autoimmune factors, neuronal plasticity, and autoimmune dysregulation. Inexplicable pain, limb edema, skin color changes, and elevated skin temperature can manifest after trauma and surgery due to an exaggerated inflammatory response [2]. Although neurogenic inflammation is a normal process after nerve injury, tissue injury in
CRPS with or without nerve injury appears to induce an exaggerated neuroinflammatory response, including the release of proinflammatory neuropeptides. Proinflammatory mediators can cause the activation of nociceptors that may lead to allodynia and hyperalgesia, which are the wellknown characteristics of CRPS that result from peripheral sensitization $[3,4]$.

As frequently observed in herpes zoster or stroke patients, many disorders of the peripheral and central nervous system cause both neuropathic itch (NI) and neuropathic pain (NP). Sensory loss and gain of function can be concomitant-

This is an Open Access article distributed under the terms of the Creative Commons Attribution Non-Commercial License (http://creativecommons.org/licenses/by-nc/4.0) which permits unrestricted non-commercial use, distribution, and reproduction in any medium, provided the original work is properly cited.

Copyright (C) the Korean Society of Anesthesiologists, 2021 
ly observed in both these conditions [5]. In NP, the hypersensitive sensory states are termed allodynia and hyperalgesia whereas the corresponding terms used in NI are alloknesis and hyperknesis. Over the past few years, studies have attempted to describe the pathophysiology of itch and its neuropathic origin. Therefore, in the light of this research, our understanding of pruritus suggests that the skin is not the only reason for the sensation of itch. Damage to the nervous system may interfere with the pruritic afferent pathways allowing painful and noxious sensations to be perceived as pruritic [5].

Although the concept of NI has been emerging, few studies have covered its pathophysiology. Unfortunately, common treatments for pruritus are often not effective for pruritus of such origins. Furthermore, NI affects a patient's quality of life to an extent similar to NP. Herein, we discuss the atypical presentation of NI in a patient with CRPS and the treatment modalities used to improve his symptoms.

\section{CASE REPORT}

We obtained written informed consent from the patient after surgery to publish this report.

A 23-year-old male presented with uncontrolled, chronic pruritus of his left foot and ankle. When he first visited the clinic, he was limping and had reduced range of motion, swelling, and sweating and flushing of the skin over the affected area. Interestingly, he was mainly concerned with the severe itching sensation and did not complain of pain. Six months before his first visit to our clinic, he had undergone open reduction and internal fixation of his left foot due to recurrent left first metatarsal fracture. To start with, the initial injury was a left great toe fracture, prompting a cast application. From the time of injury, the patient only complained of itching, rather than pain, over the affected foot. Due to the accompanying itch, he had kicked his foot against a hard wall in frustration and developed a comminuted toe fracture. Therefore, an additional surgical correction was performed. A plaster cast was applied for 3 months after the surgical correction due to the complex nature of the recurrent metatarsal fracture. Immediately after removal of the plaster cast, he developed uncontrolled pruritus and discomfort, skin color change, and swelling over the surgical site, which lasted for more than 3 months.

This patient had a history of congenital heart disease, with a functional single ventricle with transposition of the great arteries and pulmonary atresia, and had undergone three open heart surgeries by 5 years of age. At 11 days after birth, he underwent central shunt operation as his first cardiac surgery. Two weeks postoperatively, he experienced a sudden cardiac arrest for unknown reasons, and was revived. However, this had resulted in the development of ischemic brain damage (Fig. 1). The follow-up brain magnetic resonance imaging had shown multiple chronic cerebral infarctions at both the parieto-occipital lobes and left anterior watershed zone. It also showed thinning of the posterior portion of the corpus callosum and multifocal small lacunar infarctions in the left cerebellum. The ischemic stroke caused mental retardation and repeated seizures for years afterwards.

We and a consultant dermatologist did not find skin lesions could cause itching except for edema and change in skin color at the affected area. There was no change in the medication administered during the period when the patient started complaining of symptoms. In addition, itching caused by infection could be ruled out with the physical examination and normal laboratory findings. Physical examination revealed edema, change and asymmetry in skin color, sweating, and decreased range of motion at the affected foot and ankle. Moreover, he complained of severe itching when the affected foot and ankle were rubbed using a soft brush (alloknesis), and pinprick-evoked itch was also observed (hyperknesis).

Simple X-ray showed osteopenia at the affected foot and ankle. The temperature difference between both feet was up to $2.42^{\circ} \mathrm{C}$ in thermography. We found sympathetic postganglionic sudomotor dysfunction or sweat gland abnormality in the left distal leg and foot by quantitative sudomotor axon reflex test (QSART) test. In addition, a 3-phase bone scan test found diffusely increased perfusion and blood pool and delayed bone uptake in the left ankle and foot suggesting CRPS [6] (Fig. 2). Unfortunately, the energy dispersive X-ray analysis test and electrodiagnostic study were not performed because the patient did not cooperate. Although the patient did not complain of pain at all, these findings with his the symptoms and signs over the affected foot and ankle partially met the diagnostic criteria for CRPS as per the International Association for the Study of Pain (Budapest criteria) [1], and met the criteria for CRPS-NOS (not otherwise specified) [7] with itching.

We initiated his treatment carefully, with sub-anesthetic doses of intravenous (IV) ketamine therapy $(0.1-0.3 \mathrm{mg} / \mathrm{kg}$ ) administered in the outpatient pain clinic, along with prescribed oral doses of pregabalin (75 mg, twice a day) and 

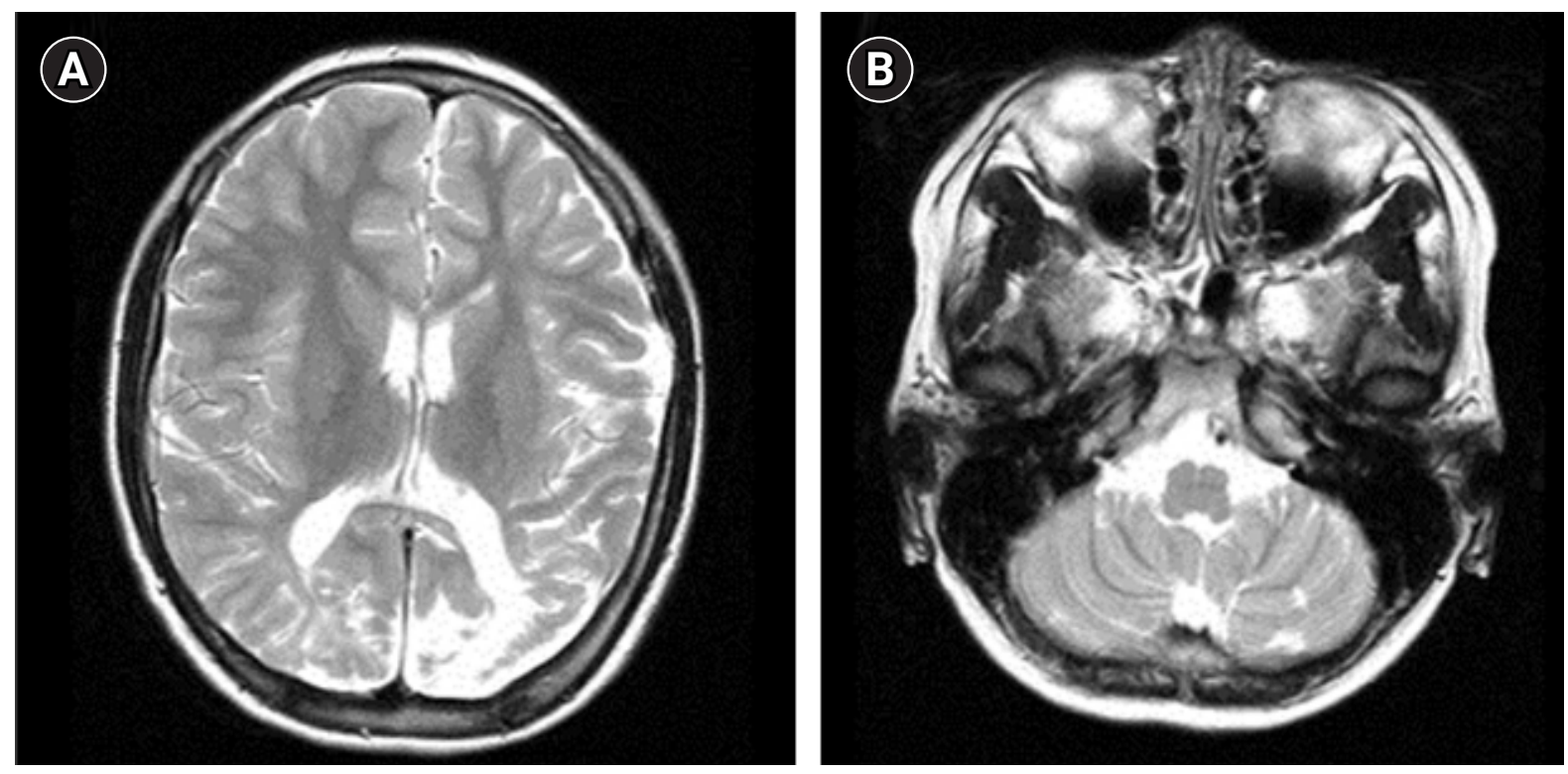

Fig. 1. Magnetic resonance imaging of the brain shows multiple chronic cerebral infarctions at both the parieto-occipital lobes and left anterior watershed zone and thinning of the posterior portion of the corpus callosum (A). Multifocal small lacunar infarctions are also seen in the left cerebellum (B).

\section{${ }^{99 \mathrm{~m}}$ Tc-DPD Whole Body Bone Scan}
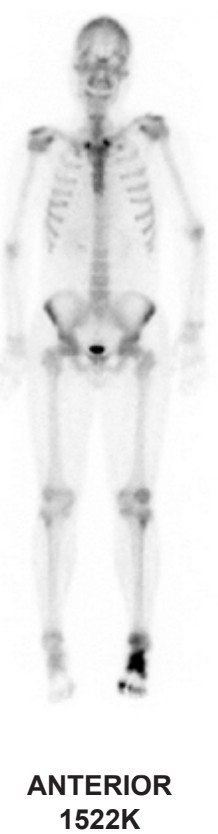

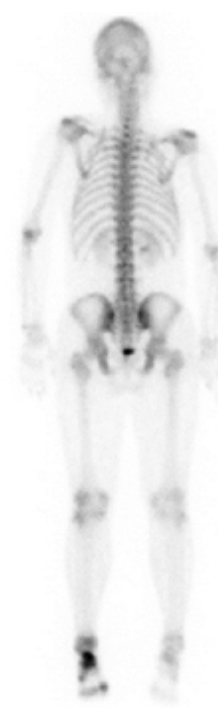

$$
\begin{gathered}
\text { POSTERIOR } \\
\text { 1458K }
\end{gathered}
$$

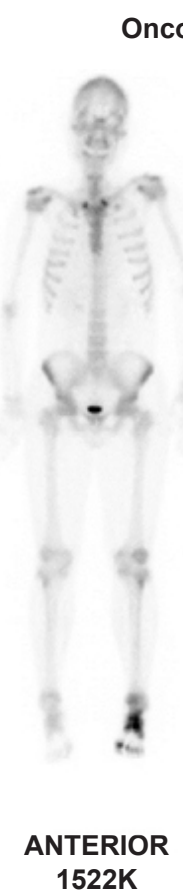

\section{Post IV 3 hrs $38 \mathrm{~min}$}

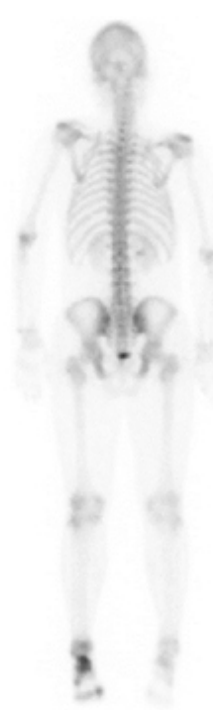

POSTERIOR $1458 \mathrm{~K}$
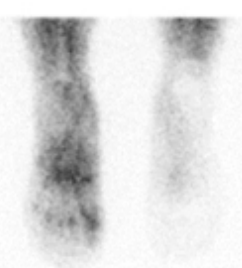

LT PLANTAR RT (1 $\mathrm{min})$

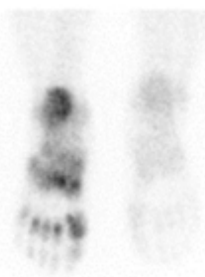

LT PLANTAR RT (delay)

Fig. 2. The three-phase bone scan shows diffusely increased perfusion, blood pool, and delayed bone uptake in the left foot and ankle. ${ }^{99 \mathrm{~m}} \mathrm{Tc}$-DPD: ${ }^{99 \mathrm{~m}} \mathrm{Tc}-3$, 3-diphosphono-1, 2-propanodicarboxylic acid, IV: intravenous. 
naproxen (500 mg, twice a day) to control his symptoms. One month later, his gait had improved, and the edema and profuse sweating had resolved. Although he experienced a few side effects, such as urinary retention and generalized edema, he could relatively tolerate them. With the same treatment every month, the pruritus improved gradually and completely subsided by the sixth month, along with the skin discoloration. Subsequently, we discontinued the IV ketamine therapy and prescribed reduced doses of pregabalin only. Therefore, the patient was successfully treated with medications used for the treatment of NP.

\section{DISCUSSION}

There could be three explanations for the questionable symptoms observed in this case. First, delayed removal of the plaster cast may have triggered the itching and skin color change. It could also have caused muscle atrophy, deep vein thrombosis, joint stiffness due to prolonged immobilization, gait abnormalities, and calf muscle weakness [8]. This could explain some of his symptoms, but they do not usually last for more than 1 month; if they do, physicians are required to look for other causes for these symptoms. Another possibility of itching was the atypical presentation of CRPS. Fracture of an extremity is a common inciting event of CRPS type 1. According to a previous report, $7 \%$ of patients with a single fracture of the wrist, scaphoid, ankle, or metatarsal bone develop CRPS type 1 [3]. With the obvious inciting events, the patient showed skin color changes, edema, and abnormal sudomotor activity, without the presence of other conditions that could account for the dysfunction. Except for the fact that the continuous pain and allodynia were substituted by continuous severe itching and alloknesis, results of the imaging test, QSART test, and 3-phase bone scan and physical evidence pointed to an atypical presentation of CRPS-NOS with itch. His successful treatment with medications used for NP supports this diagnosis. Lastly, due to his cerebrovascular disease, it is possible that the abnormal pruritus may have been caused by a lesion in the afferent sensory pathway, rather than by a cutaneous lesion or a peripheral stimulus. An unusual itch with a neurologic origin is called an NI and is defined as an itch caused by a pathology located at any point along the afferent pathway of the nervous system [5].

About $30 \%$ of patients with NI have a peripheral neuropathic cause, including post-herpetic itch, brachioradial pruritus, notalgia paresthetica, trigeminal trophic syndrome, and itch caused by burns or keloids [9]. It has been reported that $15 \%$ of itches arise from central nervous system disorders [10]. Spinal cord disorders including inflammatory transverse myelitis, neoplasms, cavernous hemangiomas, and post-traumatic Brown-Sequard syndrome have also been implicated for the same $[9,11]$. Ischemic stroke of the subcortical area or brainstem is the most common central cause of NI [10]. As shown in Fig. 1, the patient was previously diagnosed with multiple hypoxic ischemic encephalopathy. Since NI can occur in patients with central nervous system disorders, we also cannot rule out the possibility of NI due to cerebrovascular disease.

The diagnostic criteria for NI has yet to be definitively established. Currently, its diagnosis is primarily based on clinical characteristics specific to NI syndromes, with history taking as an important process to exclude dermatologic or systemic causes, as well as detect neuropathic causes [9]. Sensory tests, electrodiagnostic tests, autonomic function tests, imaging studies, and skin biopsies may also help detect and localize potential neuropathic causes [9]. In the present case, due to the patient's non-cooperation, we could not perform an electrodiagnostic test and skin biopsy; however, results from the sensory test, 3-phase bone scan, and QSART test suggested that his itching was neuropathic in origin.

Similar to the circumstances of NI diagnosis, there is also no specific treatment for this condition [9]. Generally, management of NI begins with non-pharmacological measures used for itch, followed by other therapies in a stepwise approach, wherein the choice of treatment is usually based on the size and localization of the pruritus [12,13]. In particular, treatment of itching caused by neuropathy is often based on NP management [9]. Moreover, preventing and treating secondary scratch-induced skin lesions is also important to reduce further itching and infection. Since antihistamines, corticosteroids, and most pain medications were largely ineffective in treating NI, we decided to systematically administer inhibitors of neuronal excitability, and thus the patient was treated using medications for CRPS with NP. More specifically, monthly IV sub-anesthetic ketamine doses with daily oral pregabalin and naproxen were administered to the patient for 6 months. Naproxen was prescribed in this case to reduce the release of inflammatory mediators that may affect NP and/or NI. Although he experienced a few side effects, such as urinary retention and generalized edema, the pruritus was successfully treated.

In conclusion, when a patient complains of unexplained itching not resolved by general itching treatments, a probable neuropathy should also be considered among its various 
causes. Careful history taking and evaluation of symptoms, with some neurologic and radiologic tests to detect neuropathic causes, can help identify NI. Although there is no established treatment for NI, treatment according to NP can improve these symptoms.

\section{CONFLICTS OF INTEREST}

No potential conflict of interest relevant to this article was reported.

\section{DATA AVAILABILITY STATEMENT}

All data generated or analyzed during this study are included in this published article.

\section{AUTHOR CONTRIBUTIONS}

Conceptualization: Seong-Soo Choi. Data curation: Woojong Cho, Juhan Mun, Young Do Kim. Visualization: Wonyeong Jeong. Writing - original draft: Syn-Hae Yoon. Writing - review \& editing: Woojong Cho, Juhan Mun, SeongSoo Choi. Supervision: Seong-Soo Choi.

\section{ORCID}

Syn-Hae Yoon, https://orcid.org/0000-0001-5395-0606 Woojong Cho, https://orcid.org/0000-0002-8376-5142 Juhan Mun, https://orcid.ogr/0000-0002-3228-7769 Wonyeong Jeong, https://orcid.org/0000-0002-7025-7210 Young Do Kim, https://orcid.org/0000-0003-1796-9953 Seong-Soo Choi, https://orcid.org/0000-0002-2333-0235

\section{REFERENCES}

1. Harden RN, Bruehl S, Stanton-Hicks M, Wilson PR. Proposed new diagnostic criteria for complex regional pain syndrome. Pain Med 2007; 8: 326-31.

2. Birklein F, Schlereth T. Complex regional pain syndrome-significant progress in understanding. Pain 2015; 156 Suppl 1: S94-103.

3. Urits I, Shen AH, Jones MR, Viswanath O, Kaye AD. Complex regional pain syndrome, current concepts and treatment options. Curr Pain Headache Rep 2018; 22: 10.

4. Sahbaie P, Shi X, Guo TZ, Qiao Y, Yeomans DC, Kingery WS, et al. Role of substance P signaling in enhanced nociceptive sensitization and local cytokine production after incision. Pain 2009; 145: 341-9.

5. Hachisuka J, Chiang MC, Ross SE. Itch and neuropathic itch. Pain 2018; 159: 603-9.

6. Shin SH, Kim SJ. Bone scintigraphy in patients with pain. Korean J Pain 2017; 30: 165-75.

7. Harden NR, Bruehl S, Perez RSGM, Birklein F, Marinus J, Maihofner C, et al. Validation of proposed diagnostic criteria (the "Budapest Criteria") for complex regional pain syndrome. Pain 2010; 150: 268-74.

8. Halanski M, Noonan KJ. Cast and splint immobilization: complications. J Am Acad Orthop Surg 2008; 16: 30-40.

9. Steinhoff M, Schmelz M, Szabó IL, Oaklander AL. Clinical presentation, management, and pathophysiology of neuropathic itch. Lancet Neurol 2018; 17: 709-20.

10. Dhand A, Aminoff MJ. The neurology of itch. Brain 2014; 137(Pt 2): 313-22.

11. Binder A, Koroschetz J, Baron R. Disease mechanisms in neuropathic itch. Nat Clin Pract Neurol 2008; 4: 329-37.

12. Stumpf A, Ständer S. Neuropathic itch: diagnosis and management. Dermatol Ther 2013; 26: 104-9.

13. Peerdeman KJ, Tekampe J, van Laarhoven AIM, van Middendorp H, Rippe RCA, Peters ML, et al. Expectations about the effectiveness of pain- and itch-relieving medication administered via different routes. Eur J Pain 2018; 22: 774-83. 